\title{
Transient colloid assembly by fuel-driven modulation of depletion interactions
}

\author{
Michelle P. van der Helm, ${ }^{1}$ Chang-Lin Wang, ${ }^{1}$ Reece W. Lewis, ${ }^{1}$ Sarah Schyck, ${ }^{1}$ Laura Rossi* ${ }^{1}$ and Rienk \\ Eelkema*, \\ ${ }^{1}$ Department of Chemical Engineering, Delft University of Technology, Van der Maasweg 9, 2629 HZ Delft, The Netherlands \\ KEYWORDS: Out-of-equilibrium, Chemical reaction networks, Colloids, Polymeric depletion, Assembly
}

\begin{abstract}
In biology, energy stored in chemical fuels is used to drive processes energetically uphill, enabling the highly dynamic behavior of living organisms. The out-of-equilibrium behavior can propagate from molecular reaction networks to the micro- and macroscopic scale. These natural phenomena have sparked the design of man-made out of equilibrium chemical reaction networks (CRNs) and dissipative assembly systems with hydrogels, (supra)polymers, vesicles/micelles and colloids. In colloidal systems, the assembly process is typically controlled by balancing the interaction forces. Here, we use a polymeric depletant integrated in a fuel driven esterification CRN to induce transient colloidal assembly. The polymer undergoes a temporal coil-globule transition upon acetylation by the chemical fuel. In the random coil conformation it acts as depletant agent for the silica colloids, promoting colloidal aggregation. As compact globule, the polymer loses its depletant characteristics. During the fuel cycle the polymer cyclically transitions from one form to the other, directly influencing colloidal aggregation and redispersion. Thus, a fuel-driven CRN on the molecular scale results in a microscopic response with a transient colloidal depletion cycle. Overall, the time-dependent propagation of out-of-equilibrium activity across length scales presented here, offers opportunities to design responsive materials with life like properties.
\end{abstract}

Living systems use energy in the form of chemical fuels to drive thermodynamically unfavourable processes, which gives them the ability to sense, replicate, proliferate, self-heal and amplify signals. These biological out-of-equilibrium systems have a molecular origin, yet can give rise to micro- or even macroscopic responses. ${ }^{1-3}$ Prime examples from biology are microtubules and actin filaments that play key roles in cellular motility. Their underlying CRNs are based on the consumption of a chemical fuel (i.e. GTP or ATP) to drive the assembly of transiently stable supramolecular polymers from individual activated tubulin or actin building blocks. ${ }^{4}$ Inspired by these natural systems, many dissipative assembly processes have been designed, ${ }^{5-13}$ including fuel-driven colloidal assembly ${ }^{14-19}$. The majority of these colloidal systems decorate the colloids' surface with functional groups that can then participate in a specific fuel-driven CRN. That way the interaction potential of the colloids can be altered during the fuel cycle, leading to a transient assembly response. In general, colloidal stability and assembly are governed by an interplay between different interaction forces. ${ }^{20-21}$ On top of that, nanoscale polymeric particles and surfactant assemblies can also promote colloidal assembly via depletion. ${ }^{22}$ Depletion is an attractive force of entropic origin which arises when colloids are dispersed in a solution containing non-adsorbing polymers ${ }^{23-25}$. In this scenario colloids are surrounded by a so-called exclusion zone, a volume depleted of polymers which thickness corresponds to the radius of gyration of the polymers. When two colloids approach each other so that the corresponding exclusion zones overlap (i.e. polymers cannot fit between the colloids anymore), colloids become attractive due to an osmotic pressure imbalance. The size, orientation and hydrophilicity of the depletants can be controlled by external factors, such as $\mathrm{pH}$, temperature and ionic interactions for reversible regulation of depletion interactions. ${ }^{26-32}$ In the current work, however, we aim to control colloidal interactions with a polymeric depletant that is integrated in a fuel-driven CRN. The fuel cycle integration provides autonomous control over the time domain and does not require any external temperature or $\mathrm{pH}$ changes. Previously, we developed a fuel-driven transient polymer acetylation CRN with acetic anhydride as chemical fuel to regulate product yield and lifetime. ${ }^{33}$ We were able to control polymer conformation and aggregation with two organocatalysts (pyridine and imidazole) and by varying fuel levels. The polymer we developed consisted of a poly(acrylic acid) (PAA) backbone with $25 \%$ coverage of 3-nitro-L-tyrosine (NY), which could undergo transient acetylation causing a temporal coil to globule transition. Now, we exploit this fuel-driven polymer coil-globule transition to dynamically control its depletion ability, leading to transient colloidal assembly (Figure 1). 


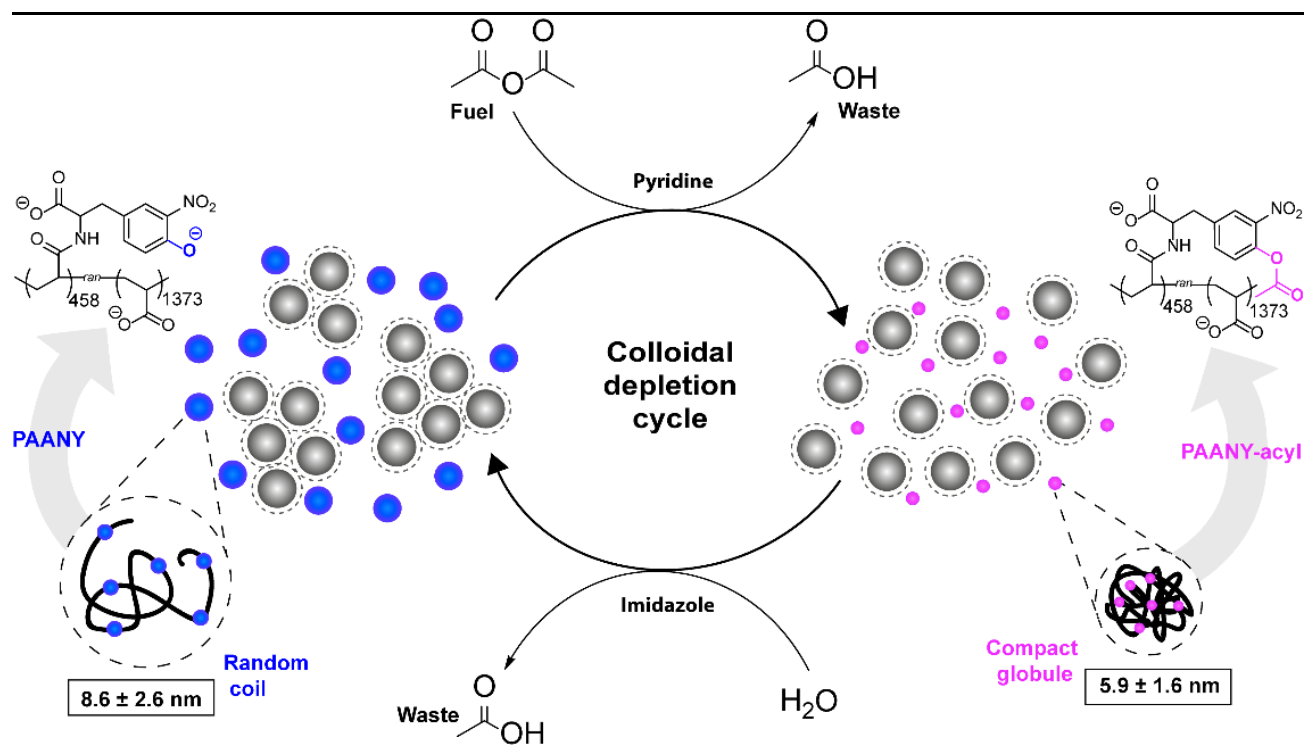

Figure 1. Colloidal depletion cycle regulated by a fuel-driven esterification network with a polymer random coil (large size depletant) to compact globule transition (small size - no depletant) due to transient acetylation of 3-nitro-L-tyrosine functionalized (poly)acrylic acid (PAANY).

PAANY with negatively charged NY groups has a random coil conformation (diameter $8.6 \mathrm{~nm}$ ). Upon addition of the chemical fuel (acetic anhydride) the NY moieties get acetylated, generating PAANY-acyl, which adopts a compact globule conformation (diameter $\sim 5.9 \mathrm{~nm}$ ) due to a decreased charged density and increased hydrophobic surface. We hypothesize that native PAANY in the random coil conformation is a polymeric depletant for silica colloids (diameter $=1 \mu \mathrm{m})$, making the colloids assemble. PAANY-acyl, as a smaller compact globule, loses the depletion capacity, leading to disassembly of the colloids. Over time, PAANY-acyl will revert back to native PAANY through hydrolysis, leading to a conformational change and a concomitant increase in depletion ability. Hence, a full fuel cycle initiated by the addition of chemical fuel on the molecular scale causes a transient colloidal assembly-disassembly-assembly cycle on the microscopic level.

At first, the depletion capability of PAANY was investigated. To this end, different concentrations of PAANY in borate buffer ( $\mathrm{pH} 9.0$, $200 \mathrm{mM}$ ) were added to a solution of silica microspheres $\left(1.15 \cdot 10^{8}\right.$ \# colloids $/ \mathrm{mL}$ ) to construct an aggregation calibration curve (Figure S1). From this curve, it becomes clear that at least $0.5 \mathrm{mM}$ PAANY should be used to have a significant depletion effect. In contrast to our previous work ${ }^{33}$, for these colloidal experiments borate buffer of $\mathrm{pH} 9.0$ was used as colloidal silica was found to stick to the glass wall of the capillary at lower $\mathrm{pH}$. In the absence of the anhydride fuel the colloids form a stable dispersion with PAANY acting as a depletant. Subsequently, different concentrations of PAANY $(0.5 / 0.6 / 0.7 \mathrm{mM})$ were used in fuel cycles with acetic anhydride (chemical fuel), pyridine (catalyst forward reaction) and imidazole (catalyst backward reaction) (Figure 2 and Figure S3-4). With lower pyridine concentration $(0.7 \mathrm{mM})$ only $50 \%$ of PAANY is acetylated at its maximum (Figure S3A - UV-VIS conversion) and the colloid (dis)assembly response is less strong (Figure S4A,B,C) compared to the optimized conditions (Figure 2A and Figure S4D). With more pyridine present $(1.75 \mathrm{mM})$ almost $90 \%$ of the NY groups on the polymer are acetylated (Figure S3B - UV-VIS conversion) and the colloidal assembly cycle is more pronounced (Figure 2A and Figure S4D). In Figure 2A the colloid aggregation (\%) as a function of time is shown for the optimized conditions ( $0.7 \mathrm{mM}$ PAANY with 2.5 equivalents of pyridine and imidazole). Initially, the colloids are $55 \%$ aggregated due to effective depletion interactions with PAANY. After addition of 20 equivalents of the anhydride fuel, the colloid clusters immediately disassemble and an aggregation of only $30 \%$ can be observed with mostly dimer structures present (Figure $2 \mathrm{C}$ - microscopy image $5 \mathrm{~min}$ ). Hence, the polymer in its globule state with is not able to deplete the colloids anymore at this concentration and the large clusters disassemble. During the fuel cycle the colloidal aggregation increases again and microscopy images after $90 \mathrm{~min}$ and 180 min show the reappearance of the larger colloidal clusters (Figure $2 \mathrm{D}, \mathrm{E}$ ). This can be attributed to an increase in the depletion interactions as the polymer is deacetylated back and in its random coil conformation again. The colloidal clusters from the reference microscopy images of the starting sample can be viewed in Figure S5 (high and low magnification), overall corresponding to an average aggregation percentage of $54.5 \pm 5.78 \%$. Microscopy images of colloidal assembly over the course of a full fuel cycle are presented in Figures S6,7 (high and low magnification). 


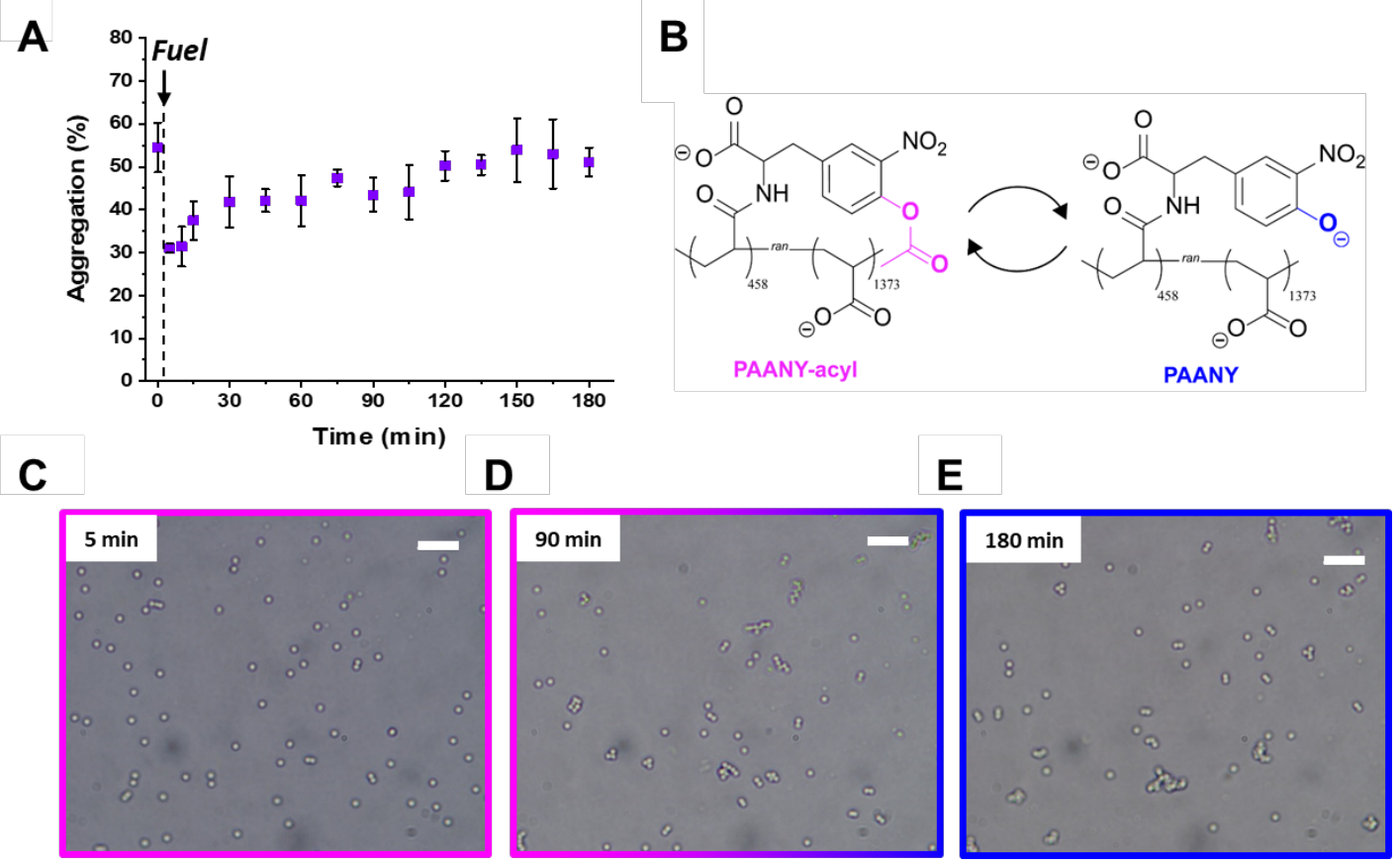

Figure 2. (A). Colloid aggregation as a function time for a fuel cycle. (B). Upon fuel (acetic anhydride) addition PAANY is acetylated to PAANY-acyl. (C). The acetylation induces colloidal disassembly (30\% aggregation at $5 \mathrm{~min})$. (D-E). Over time PAANY-acyl is deacetylated giving rise to the reappearance of colloidal clusters ( $40 \%$ aggregation at $90 \mathrm{~min}$ and $50 \%$ aggregation at $180 \mathrm{~min}$ ). Conditions: PAANY $0.7 \mathrm{mM}$, pyridine $1.75 \mathrm{mM}$, imidazole $1.75 \mathrm{mM}$, acetic anhydride $14 \mathrm{mM}$ in borate buffer $(\mathrm{pH} 9.0200 \mathrm{mM})$ with silica microspheres (diameter= $1 \mu \mathrm{m})$. The standard deviation between two samples is shown $(\mathrm{n}=2)$. The point measured at $\mathrm{t}=0 \mathrm{~min}$ is a different sample without fuel addition, but all other conditions the same. The scale bar in all images is $10 \mu \mathrm{m}$. The aggregation (\%) was determined by using Matlab.

Next to the overall aggregation over time, the distribution over the various cluster sizes was evaluated (Figure 3 ). As apparent from Figure 3 , the percentage of dimer structures (Figure 3A) remains fairly constant over the course of the fuel cycle. On the contrary, the contribution of trimers (Figure 3B) and especially larger clusters $(>3$ particles) (Figure 3C) changes over time and follows the trend of the overall aggregation. Larger clusters are present from the start and disappear completely upon fuel addition, while over time they slowly reassemble. The cluster structures that we observe for all samples are not entirely crystalline, but show a fractal-like open structure (Figure 3D). We hypothesize that this is due to the existence of a secondary minimum in the interaction potential. The formation of the fractal structures is most likely caused by an increased depletion attraction force and hence reduced probability of escape for a particle in a cluster. This phenomenon has also been observed in other research with PAA polymers used for depletion-flocculation studies of polystyrene latex particles ${ }^{34-35}$ and silica surfaces ${ }^{36}$. The secondary minimum is also quite shallow, as corroborated by the presence of single particles alongside the larger clusters (Figure 3D). Another reason for the presence of open structures can be polymer adsorption onto the colloid surface. However, adsorption here can be ruled out since it usually happens for PAA polymers having protonated carboxylates at low $\mathrm{pH}^{34,36}$ Furthermore, the mobility of the particles is not influenced by the fuel cycle. We confirmed this by calculating the mean squared displacement (MSD) as a function of time (see SI section Mean squared displacement from particle tracking).

In this work, we have shown how polymer conformational changes regulated by a fuel-driven esterification $\mathrm{CRN}$ can induce transient colloidal depletion aggregation. Upon acetylation by a chemical fuel, the polymer undergoes a temporal transition from a random coil to a compact globule. In the random coil conformation it acts as a polymeric depletant for silica colloids, while in its globular form it is too small to act as depletant for this system. Later in the fuel cycle the polymer is deacetylated again, reobtaining its depletion capacity. The concept was visualized using optical microscopy. The colloidal clusters are fractal-like structures due to the existence of a secondary minimum and the colloids remain dynamic over the course of the fuel cycle. Overall, a fuel cycle initiated by the addition of chemical fuel on the molecular scale results in a microscopic response with a transient colloidal depletion cycle and thus provides a temporary way out of thermodynamic equilibrium on large length scales. Understanding the propagation from a molecular reaction network to the microscopic or even macroscopic world is important for further research in this field and can support the design of responsive materials with life-like properties. 
A
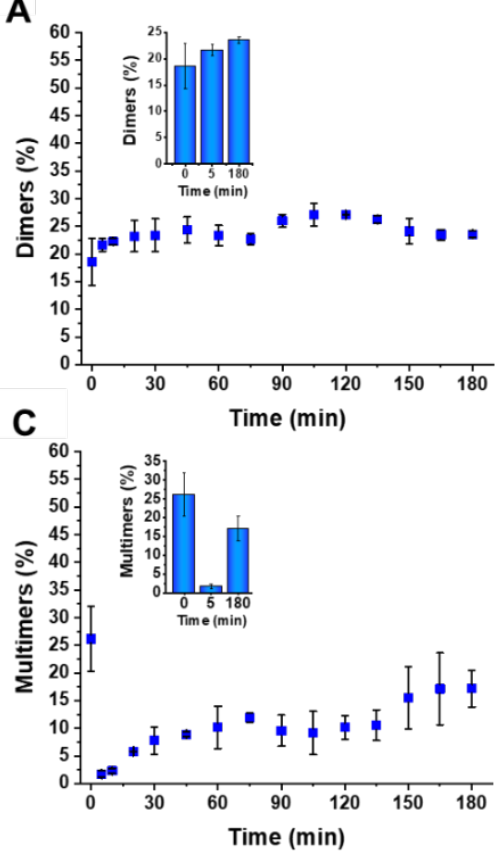

B
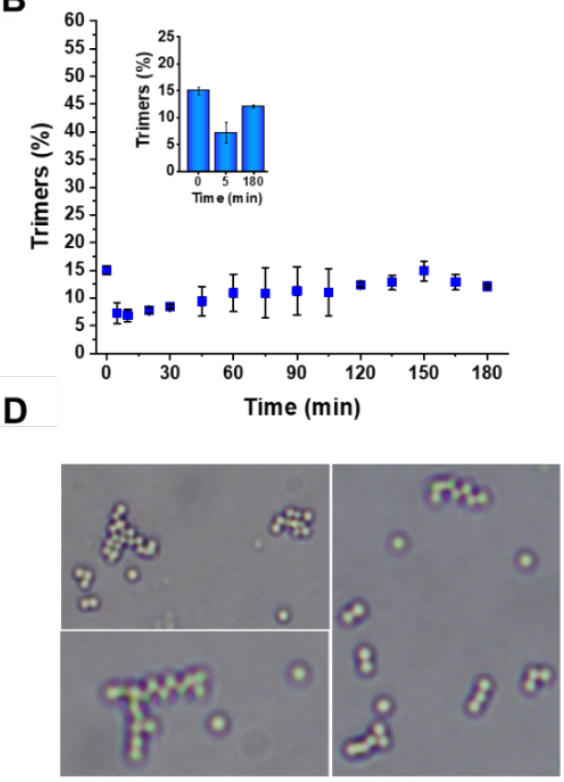

Figure 3. Cluster distribution over time: (A). Dimers contribution. (B). Trimers contribution. (C). Multimer (>3 particles) contribution. (D). Representative image of multimer fractal structures. Conditions: PAANY $0.7 \mathrm{mM}$, pyridine $1.75 \mathrm{mM}$, imidazole $1.75 \mathrm{mM}$, acetic anhydride $14 \mathrm{mM}$ in borate buffer ( $\mathrm{pH} 9.0200 \mathrm{mM}$ ) with silica microspheres (diameter=1 $\mu \mathrm{m}$ ). The standard deviation between two samples is shown ( $\mathrm{n}=2)$. The point measured at $\mathrm{t}=0 \mathrm{~min}$ is a different sample without fuel addition, but all other conditions the same. The bar charts inside the plots highlight the starting point $(0 \mathrm{~min})$, the start of fuel addition $(5 \mathrm{~min})$ and the endpoint $(180 \mathrm{~min})$.

\section{ASSOCIATED CONTENT}

\section{Supporting Information.}

Experimental details, supplemental figures referred to in the main text obtained with different techniques (PDF).

Video of colloidal particles at reference $t=0 \mathrm{~min}$, both at high and low magnification

Video of colloidal particles in fuel cycle at $\mathrm{t}=5 \mathrm{~min}$, both at high and low magnification

Video of colloidal particles in fuel cycle at $\mathrm{t}=180 \mathrm{~min}$, both at high and low magnification

"This material is available free of charge via the Internet at http://pubs.acs.org."

\section{AUTHOR INFORMATION}

\section{Corresponding Authors}

*R.Eelkema@tudelft.nl

*L.Rossi@tudelft.nl

\section{Author Contributions}

M.P.v.d.H and C-L.W. carried out the experiments and analyzed the data. R.L. and S.S. developed the Matlab particle counting and tracking scripts. L.R. provided suggestions on experiments, analysis and improvements. M.P.v.d.H, C-L.W, L.R. and R.E. designed the experiments. M.P.v.d.H. wrote the manuscript. R.E. and L.R. conceived and directed the overall research project and revised the manuscript. All authors commented on the work and the manuscript.

\section{Funding Sources}

Generous funding by the European Research Council (ERC consolidator grant 726381) is acknowledged.

\section{Notes}

The authors declare no competing financial interest.

\section{ABBREVIATIONS}

CRN, Chemical reaction network; PAANY, (poly)acrylic acid 3-nitroL-tyrosine; ATP, Adenosine triphosphate; GTP, Guanosine triphosphate.

\section{REFERENCES}

1. Bialek, W., Biophysics: searching for principles. Princeton University Press: 2012

2. Branscomb, E.; Biancalani, T.; Goldenfeld, N.; Russell, M., Escapement mechanisms and the conversion of disequilibria; the engines of creation. Phys. Rep. 2017, 677, 1-60.

3. Gnesotto, F.; Mura, F.; Gladrow, J.; Broedersz, C. P., Broken detailed balance and non-equilibrium dynamics in living systems: a review. Rep. Prog. Phys. 2018, 81 (6), 066601.

4. Alberts, B.; Johnson, A.; Lewis, J.; Morgan, D.; Raff, M.; Roberts, K.; Walter, P., Molecular biology of the cell. Sixth edition ed.; Garland Science: New York, 2014.

5. van Rossum, S. A. P.; Tena-Solsona, M.; van Esch, J. H.; Eelkema, R.; Boekhoven, J., Dissipative out-of-equilibrium assembly of man-made supramolecular materials. Chem. Soc. Rev. 2017, 46 (18), 5519-5535.

6. Singh, N.; Formon, G. J.; De Piccoli, S.; Hermans, T. M., Devising Synthetic Reaction Cycles for Dissipative Nonequilibrium Self-Assembly. Adv. Mater. 2020, 32 (20), 1906834.

7. Boekhoven, J.; Hendriksen, W. E.; Koper, G. J. M.; Eelkema, R.; van Esch, J. H., Transient assembly of active materials fueled by a chemical reaction. Science 2015, 349 (6252), 1075-1079.

8. Leira-Iglesias, J.; Tassoni, A.; Adachi, T.; Stich, M.; Hermans, T. M., Oscillations, travelling fronts and patterns in a supramolecular system. Nat. Nanotechnol. 2018, 13 (11), 1021.

9. Ragazzon, G.; Prins, L., Energy consumption in chemical fuel-driven self-assembly. Nat. Nanotechnol. 2018, 13, 882-889.

10. Heuser, T.; Weyandt, E.; Walther, A., Biocatalytic feedback-driven temporal programming of self-regulating peptide hydrogels. Angew. Chem. Int. Ed. 2015, 54 (45), 13258-13262.

11. Dhiman, S.; Jain, A.; Kumar, M.; George, S., Adenosine-phosphatefueled, temporally programmed supramolecular polymers with multiple transient states. J. Am. Chem. Soc. 2017, 139 (46), 16568-16575. 
12. Debnath, S.; Roy, S.; Ulijn, R. V., Peptide nanofibers with dynamic instability through nonequilibrium biocatalytic assembly. J. Am. Chem Soc. 2013, 135 (45), 16789-16792.

13. Sorrenti, A.; Leira-Iglesias, J.; Sato, A.; Hermans, T. M., Nonequilibrium steady states in supramolecular polymerization. Nat. Comm. 2017, 8, 15899 .

14. van Ravensteijn, B. G.; Voets, I. K.; Kegel, W. K.; Eelkema, R., Outof-equilibrium colloidal assembly driven by chemical reaction networks. Langmuir 2020, 36, 10639-10656.

15. van Ravensteijn, B. G.; Hendriksen, W. E.; Eelkema, R.; van Esch, J. H.; Kegel, W. K., Fuel-mediated transient clustering of colloidal building blocks. J. Am. Chem. Soc. 2017, 139 (29), 9763-9766.

16. Grötsch, R. K.; Ang1, A.; Mideksa, Y. G.; Wanzke, C.; Tena-Solsona, M.; Feige, M. J.; Rieger, B.; Boekhoven, J., Dissipative Self-Assembly of Photoluminescent Silicon Nanocrystals. Angew. Chem. Int. Ed. 2018, 57 (44), 14608-14612.

17. Grötsch, R. K.; Wanzke, C.; Speckbacher, M.; Angı, A.; Rieger, B.; Boekhoven, J., Pathway dependence in the fuel-driven dissipative selfassembly of nanoparticles. J. Am. Chem. Soc. 2019, 141 (25), 9872-9878.

18. Heuser, T.; Steppert, A.-K.; Molano Lopez, C.; Zhu, B.; Walther, A., Generic concept to program the time domain of self-assemblies with a selfregulation mechanism. Nano Lett. 2015, 15 (4), 2213-2219.

19. Dehne, H.; Reitenbach, A.; Bausch, A., Transient self-organisation of DNA coated colloids directed by enzymatic reactions. Sci.Rep. 2019, 9 (1), $1-9$.

20. Russel, W. B.; Russel, W.; Saville, D. A.; Schowalter, W. R., Colloidal dispersions. Cambridge university press: 1991.

21. Evans, D. F.; Wennerström, H., The colloidal domain: where physics, chemistry, biology, and technology meet. 1999.

22. Tuinier, R.; Lekkerkerker, H. N., Colloids and the Depletion Interaction. Springer Netherlands: 2011.

23. Asakura, S.; Oosawa, F., Interaction between particles suspended in solutions of macromolecules. J. Polym. Sci. 1958, 33 (126), 183-192.

24. Asakura, S.; Oosawa, F., On interaction between two bodies immersed in a solution of macromolecules. J. Chem. Phys. 1954, 22 (7), 1255-1256.
25. Mao, Y.; Cates, M.; Lekkerkerker, H., Depletion force in colloidal systems. Physica A 1995, 222, 10-24.

26. Buzzaccaro, S.; Colombo, J.; Parola, A.; Piazza, R., Critical depletion. Phys. Rev. Lett. 2010, 105 (19), 198301.

27. Piazza, R.; Buzzaccaro, S.; Parola, A.; Colombo, J., When depletion goes critical. J. Condens. Matter Phys. 2011, 23 (19), 194114.

28. Liu, Z.; Niu, Z.-w., Temperature responsive 3D structure of rod-like bionanoparticles induced by depletion interaction. Chinese J. Polym. Sci. 2014, 32 (10), 1271-1275.

29. Lele, B. J.; Tilton, R. D., Control of the colloidal depletion force in nonionic polymer solutions by complexation with anionic surfactants. $J$. Colloid Interface Sci. 2019, 553, 436-450.

30. Xing, X.; Li, Z.; Ngai, T., pH-controllable depletion attraction induced by microgel particles. Macromolecules 2009, 42 (19), 7271-7274.

31. Gratale, M. D.; Still, T.; Matyas, C.; Davidson, Z. S.; Lobel, S.; Collings, P. J.; Yodh, A., Tunable depletion potentials driven by shape variation of surfactant micelles. Phys. Rev. E 2016, 93 (5), 050601.

32. Feng, L.; Laderman, B.; Sacanna, S.; Chaikin, P., Re-entrant solidification in polymer-colloid mixtures as a consequence of competing entropic and enthalpic attractions. Nat. Mater. 2015, 14 (1), 61-65.

33. van der Helm, M. P.; Wang, C.-L.; Fan, B.; Macchione, M.; Mendes, E.; Eelkema, R., Organocatalytic control over a fuel-driven transient esterification network, Angew. Chem. Int. Ed. 2020 DOI:10.1002/anie.202008921.

34. Burns, J. L.; Jameson, G. J.; Biggs, S., Relationship between interaction forces and the structural compactness of depletion flocculated colloids. Colloids Surf. A Physicochem. Eng. Asp. 2000, 162 (1-3), 265-277.

35. Liang, W.; Tadros, T. F.; Luckham, P., Flocculation of sterically stabilized polystyrene latex particles by adsorbing and nonadsorbing poly (acrylic acid). Langmuir 1994, 10 (2), 441-446.

36. Milling, A. J.; Vincent, B., Depletion forces between silica surfaces insolutions of poly (acrylic acid). J. Chem. Soc. Faraday Trans. 1997, 93 (17), 3179-3183.

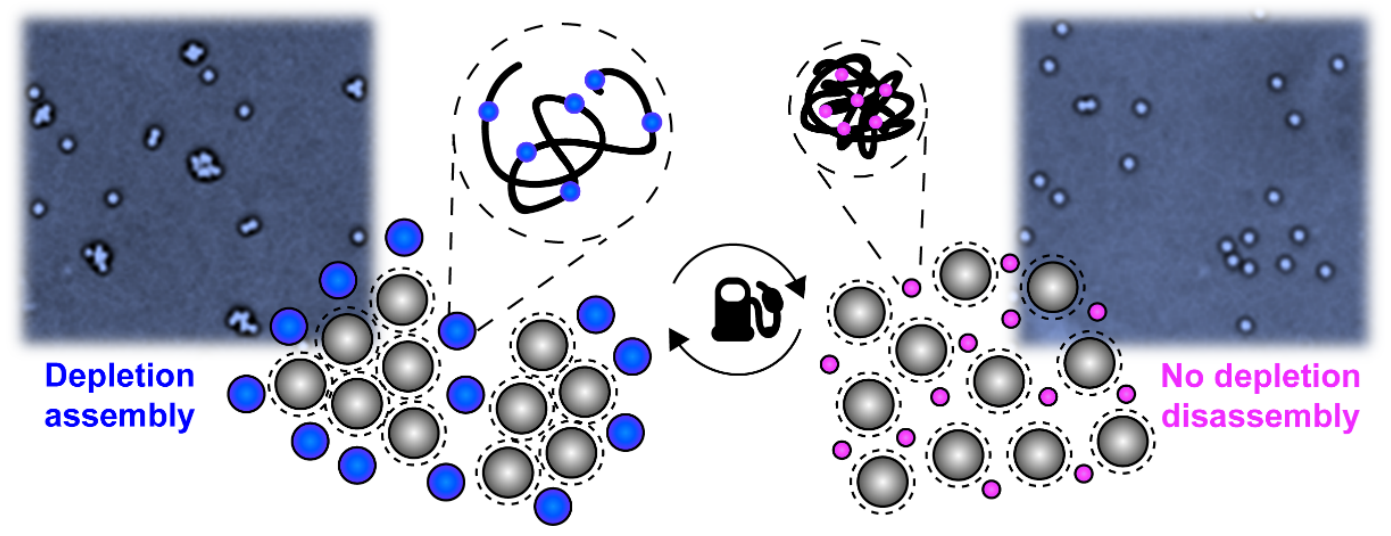

